\title{
Applying virtual reality to remote control of mobile robot
}

\author{
Chin-Shan Chen ${ }^{1, *}$ and Ching-Wen Lui ${ }^{2}$ \\ ${ }^{1}$ National Pingtung University of Science and Technology, No. 1, Shuefu Road, Neipu, Pingtung,91201, TAIWAN R.O.C. \\ ${ }^{2}$ Van Guard Mold Enterprise CO. LTD., No.11, Dazhan Ln, Yanchao Dist., Kaohsiung City, 82444, TAIWAN R.O.C.
}

\begin{abstract}
The purpose of this research is based on virtual reality to assisted pick and place tasks. Virtual reality can be utilized to control remote robot for pick and place element. The operator monitored and controlled the situation information of working site by Human Machine Interface. Therefore, we worked in harsh or dangerous environments that thing can be avoided. The procedure to operate mobile robot in virtual reality describes as follow: An experiment site with really experimental equipment is first established. Then, the experimental equipment and scene modeling are input to virtual reality for establishing a environment similar to the reality. Finally, the remote mobile robot is controlled to operate pick and place tasks through wireless communication by the object operation in virtual reality. The robot consists of a movable robot platform and robotic arm. The virtual reality is constructed by EON software; the Human Machine Interface is established by Visual Basic. The wireless connection is equipped the wireless Bluetooth, which is set the PC and PLC controller. With experimental tests to verify the robot in virtual reality and the wireless remote control, the robot could be operated and controlled to successfully complete pick and place tasks in reality by Human Machine Interface.
\end{abstract}

\section{Introduction}

Virtual Reality (VR) has been widely applied to various industries with the development of science and technology, such as education, entertainment, military affairs, space science, industrial design, and manufacturing, etc. Playing a important role in such domains, VR integrates the machinery and becomes the development of human machine interface (HMI). VR is often applied to aided instruction, training, or simulation of manufacturing process and restoring the real scenes. In addition to excluding the restrictions of location and time, it could reduce costs and avoid damage for people and machine.

The popularity of automation has resulted in many factory largely replacing manpower with robots. Mobile robots are expected to enhance the ability of the users in executing complex work in a remote environment $[1,2]$. However, wrong operation would still occur when robots are set or operated by manpower that danger and machinery breakdown could be caused. For this reason, training with VR replacing the real operation training could reduce the costs resulted from accident and machinery breakdown.

Furthermore, the development costs for a real prototype is very high, and the pick and place process could not be repeatedly applied that pick and place presents equal importance to the design and manufacturing process $[3,4]$. For pick and place tasks, the aid of reality could reduce errors and quickly completing the work. Two issues are required for simulating manufacturing systems with computers, namely simulation of non-continuous events and Virtual Reality software[5]. During the remote operation, the awareness of the remote environment is primary for an operator. With remote presentation, an operator could receive the information from the remote environment that favorable object modeling is required for simulating the object behaviors in Virtual Reality.

This study expects to applied the interactive VR to having the users rapidly and clearly understand the environment and the operation, implementing the simulation of robot operation in the actual environment, and operate and control the mobile robot in the reality through wireless remote monitoring and control so as to successfully complete the pick and place tasks by a robot.

\section{Experimental system structure}

The experimental system structure for controlling mobile robot proceeding pick and place with the integration of VR and remote control is explained as follows.

The hardware for the system contains robot arm, mobile robot, wireless Bluetooth, PC, and Programmable Logic Controller (PLC). The model for the robot arm with two degrees of freedom is mechanism for pick and place tasks. Besides, design an electromagnet for pick and place tagger. The model for the mobile robot platform is mobile car. The model for the wireless Bluetooth is HL-MD08P-PLC-C1(v2.1+EDR). PC is used for the control, which is equipped with Intel Corei5-4670 CPU 3.40GHz processing unit, Windows 7

Corresponding author: chencs@mail.npust.edu.tw 
operating system, 4G memory (RAM), and Intel HD Graphics 4600 NVIDIA GeForce GT 610 graphics card. Fig. 1 Shows that software for the system contains Pro/ENGINEER Wildfire 2.0, 3D Studio Max 7, EON Studio 5.2 (EON Studio is a development tools in VR which supplier is EON Reality company.), Visual Basic 6.0 and GX Developer 8. Physical property is included in EON, functions to present the physical properties of an object, such as physical collision, friction, and gravity effect, so as to vivify the objects in VR. The solid graphic module of robot arm and mobile robot are constructed with Pro/ENGINEER software and transferred into 3D Studio Max software for being edited Then, transferred into .eoz file with EON Trans software for VR being edited in EON Studio. This HMI of the remote control programming software in this study is designed by Visual Basic. The control program for mobile robot and arm is wrote by GX Developer. A PC is used for connecting with Wireless Bluetooth.

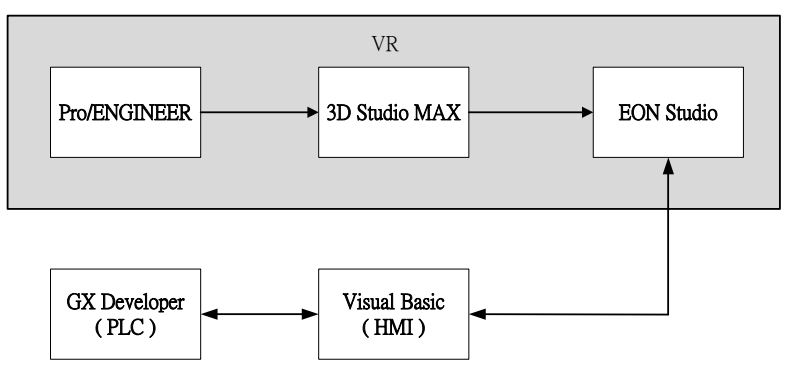

Fig. 1. Software system.

\section{Virtual reality}

VR is defined as the science integrating human and information. In other words, it is the $3 \mathrm{D}$ and interactive environment created by a computer, standing for a real world or an imagined virtual environment.

For Virtual Reality, 3D is an essential object for simulating a real world. Being a virtual environment constructed by computer graphics, Virtual Reality allows the users experiencing the real world [6] that they could observe an object according their demands, rather than being restricted in the defined objects. With the example of stereoscopic puzzles, learners could observe the positions of puzzles by changing the angle of view in Virtual Reality. It is considered being closer to problemsolving in the real world, without being restricted in certain solution.

The Robot Association started studying the applications of Virtual Reality in 1990 and regarded the great potential of Virtual Reality for Human-Machine Interface [5]. The applications of Virtual Reality have expanded the remote monitoring domain for human beings $[7,8]$. With the effects of computer computing speed and network transmission rate, the early research could merely transmitted limited information and uncontinuous frames. To overcome the delay problem, robots and the $3 \mathrm{D}$ interface for the work environment were utilized for remote operation, providing an operator with real scenes for replacing the delayed images [9]. Considering the applications of robotic arms, 2D plane displays could not satisfy the high-degree of freedom operates of robot arms. 3D graphs therefore is applied for displaying various information to better correspond to an operator's demands [10]. The renewal and update of robot hardware allow real-time addition and change in Virtual Reality. Having 3D virtual scenes to present the on-site information, present problem of insufficient bandwidth could be solved, as 3D virtual scenes offer several angles of view, which could better provide an operator with operation information, comparing to a camera shooting from fixed angles [11].

Virtual Reality is also well developed in other fields, like medical rehabilitation[12,13], simulation of surgery, driving simulation of large vehicles, virtual manufacturing process of various products and materials, virtual operation of arms in distinct military affairs, virtual training of a soldier, and virtual cities [14], and virtual training process of education [15].

To have identical motions of virtual objects and real objects, the translation and rotation of a real object should be understood. The control of virtual object is also described as follows. Fig. 2 shows the coordinates and the rotation of virtual objects in various angles, including Roll, Pitch, and Heading. In consideration of the control being the key in dynamic mechanical design, virtual simulation should present the same concept[16]. For this reason, the action and overall moving procedure of a real object should be actually realized in advance so as to establish the control of virtual objects in EON.

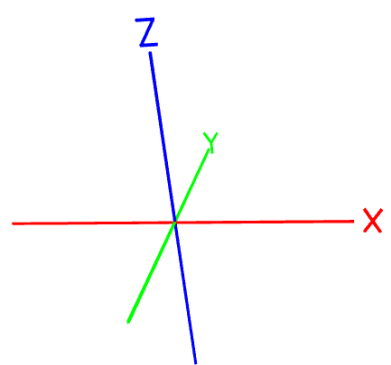

(a)

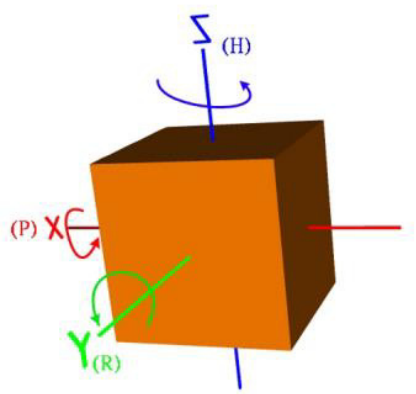

(b)

Fig. 2. (a) Definition the coordinates of EON; (b) Definition the Roll, Pitch, and Heading of EON.

With the example of translation control of a mobile robot in VR, the explanations of the translation control is 
as follows. To finish the translation of mobile robot is setting by the place node which in simulation tree of EON as in Fig. 3. First, open the node of place in EON. Second, key in the parameters for forward and click icon of the type coordinates. Then, the switch of communication for HMI is setting in the 'InEvent' as in Fig. 4. Finally, we adjusted the value of the parameters to match the mobile robot in reality. Such judgments could control the direction of mobile robot; besides, the parameters for forward, backward, left turn, right turn, and distance movement are set, which are respectively operated by click on the push button graphic $\lceil\mathrm{FOR}\rfloor$,

$\lceil\mathrm{REV}\rfloor,\lceil\mathrm{TURN} \mathrm{L}\rfloor$, and $\lceil\mathrm{TURN} \mathrm{R}\rfloor$ and $\lceil\mathrm{STOP}\rfloor$ on the HMI as in Fig. 5. Fig. 6 shows the HMI with EON in start status. The adjustment of damping parameter could change the speed of module motion.

\begin{tabular}{|c|c|c|c|c|c|c|}
\hline SimulationTree & Settings & & & & & \\
\hline Simulation & -Movement & & - Time to mo & & & \\
\hline 1.9. Scene & $\mathrm{x}:[0$ & [m] & $\mathrm{x}: 0$ & [s] & RelX & - $\mathrm{AbSX}$ \\
\hline 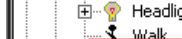 & $y: 0$ & {$[\mathrm{~m}]$} & $y: 0$ & [s] & C RelY & - AbsY \\
\hline$\Rightarrow$ Place & $z: \longdiv { 0 }$ & [m] & $z: 0$ & [s] & $C \operatorname{RelZ}$ & - $\mathrm{Abz} Z$ \\
\hline 由... Amblent & $h: 0$ & [deg] & h: 0 & [s] & $C \mathrm{RelH}$ & - $\mathrm{AbsH}$ \\
\hline It Yiewports & $\mathrm{p}: 0$ & [deg] & $\mathrm{p}: 0$ & [s] & $C$ RelP & - $\mathrm{AbsP}$ \\
\hline & $\mathrm{r}: \overline{0}$ & [deg] & $\mathrm{r}: 0$ & [s] & $C$ RelR & - $A b s R$ \\
\hline & $\begin{array}{l}\text { Active } \\
C \text { Yes }\end{array}$ & No & & & & \\
\hline & & & 確定 & & 取消 & 尞用 \\
\hline
\end{tabular}

Fig. 3. Setting the node of place in EON.

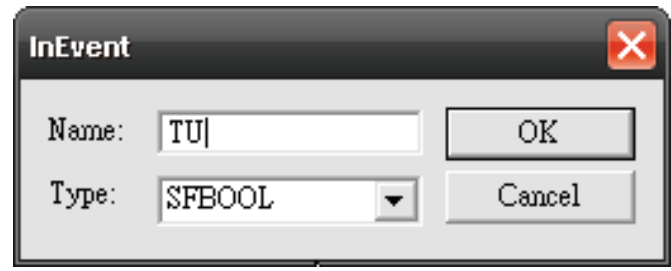

(a)

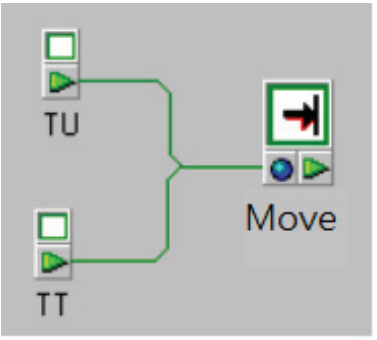

(b)

Fig.4. (a) Create an external communication event; (b) Setting the node of switch in EON.

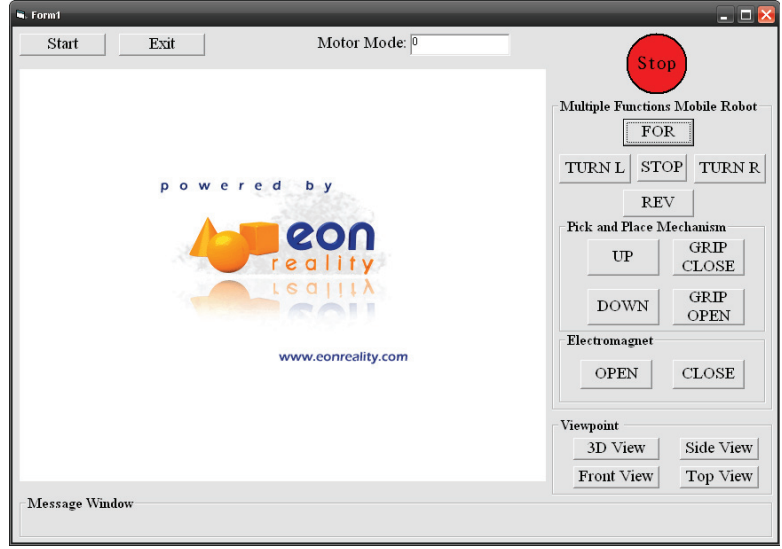

Fig. 5. HMI with EON.

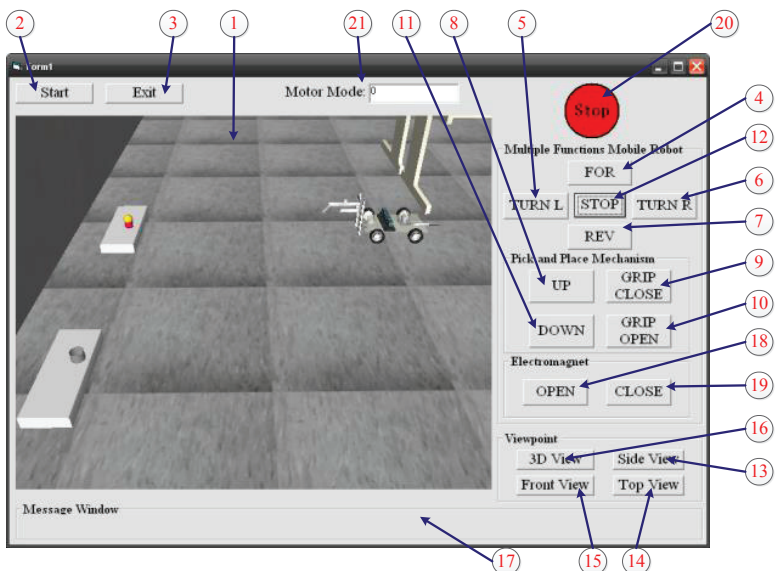

Fig. 6. HMI with EON in start status.

\section{Virtual reality integrated mobile robot control}

The virtual scenes are first input to the module in EON, simulation is used for setting the control parameters of objects, and mobile robot and communication framework are further installed. The robot test is then proceeded in VR, as the following procedure.

\subsection{Control procedure}

An indoor solid experiment is first setup, which includes mobile robot, robot arm, work platform, and communication framework. A similar scene is also established in VR to verify the synchronous operation of the robot. In the experimental scene, a mobile robot equipped with robot arm and two platforms are established; No. 2 platform is equipped with object and No. 3 platform is equipped a hole with round shape. The robot is controlled to walk a straight line from No. 1 to No.2 platform, takings the object and turning 90 degree to No. 3 platform, place the object in the hole with the same shape, and return to the start point, Fig. 7. 


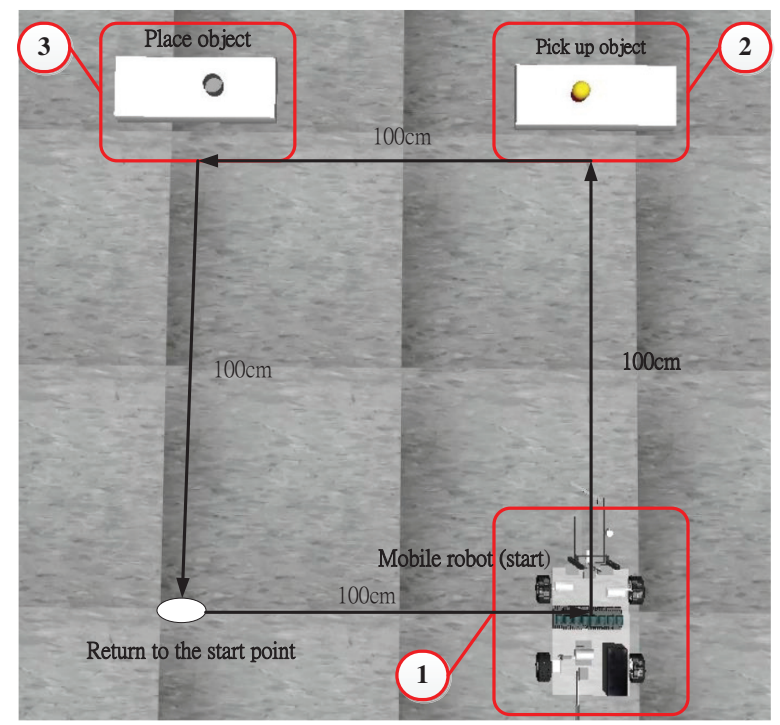

Fig. 7. Control procedure.

\subsection{Connection of robot}

The connection is proceeded with Bluetooth; then, the robot motion, which is originally controlled by push button under PLC based, is called out the connection function in windows by switch node in EON. The synchronous connection control could be operated through VR scenes. Fig. 8 shows a HMI, VR relationship with reality of mobile robotic control system. The wireless communication control utilizes the wireless transmission specifications and standards of IEEE 802.15.1 bandwidth.

\subsection{Operate and control of mobile robot}

The mobile robot motion mentioned in section 3 is controlled by click on the push button graphic 「FOR」,

$\lceil\mathrm{REV}\rfloor,\lceil\mathrm{TURN} \mathrm{L}\rfloor$, and $\lceil\mathrm{TURN} \mathrm{R}\rfloor$ and $\lceil\mathrm{STOP}\rfloor$ on the HMI. The control of robotic arm for pick and place mechanism is similar to it of wheels. Robotic arm, on the other hand, are divided and controlled by 「UP」 for moving up and 「DOWN」 for moving down for pick and place mechanism, $\lceil$ GRIP CLOSE $\rfloor$ for operating close and $\lceil$ GRIP OPEN $\rfloor$ for operating open for gripper, 「OPEN $\rfloor$ for generating the magnetic force and $\lceil\mathrm{CLOSE}\rfloor$ for not to generate the magnetic force an electromagnet, as shown in Fig. 9. We can set the different viewpoint in the human-machine interface. The user can select the different viewpoint to watch a mobile robot in VR, Such as, 「3D View」, 「Side View」, and 「Front View」.

The mobile robot and arm, gripper could simultaneously move without any effects. However, simultaneously moving forward and backward at the same part would not appear any motions.

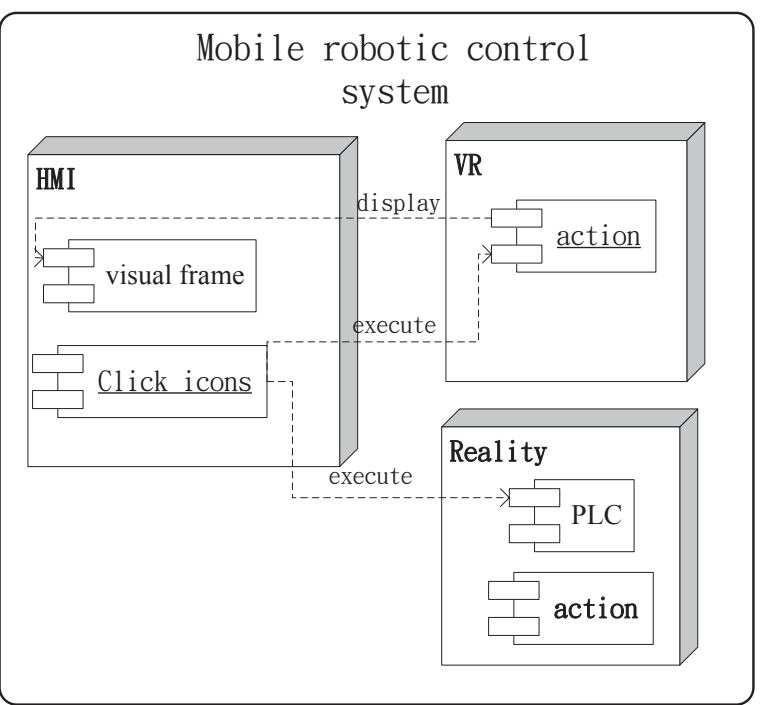

Fig. 8. Mobile robotic control system.

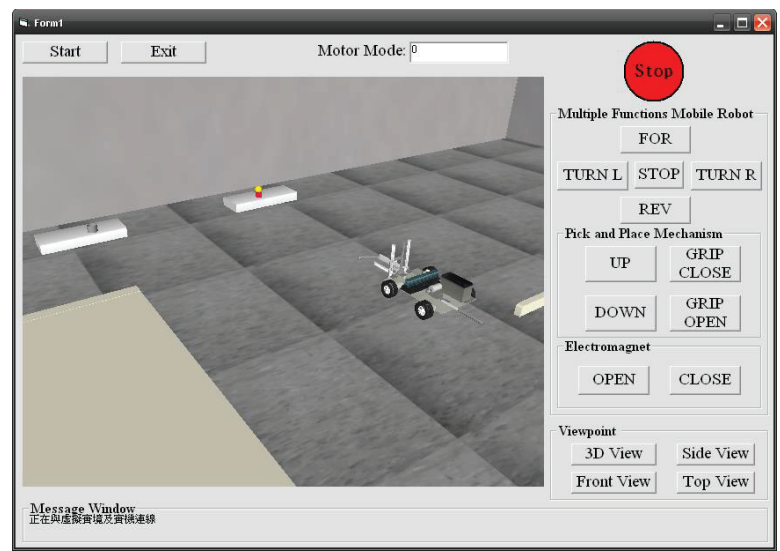

Fig. 9. To operate and control the mobile robot.

\subsection{Experimental results and discussions}

The mobile robot is first controlled to move to No. 2 work platform, and the clamp of robotic arms with pick and place mechanism is then controlled to the top of the plug-in for grabbing the plug-in by opening the clamp of robotic arms and operating the clamp to the proper position. The mobile robot is further moved to No. 3 work platform for the assembly of plugging the plug-in to the plug with the same shape, and then returned to the start point.

With the experimental test, the mobile robot forward speed appears $0.5804 \mathrm{~m} / \mathrm{sec}$ in reality, while the virtual mobile robot forward speed being adjusted the speed parameter reveals $77.84 \mathrm{~m} / \mathrm{sec}$, about $0.5804 \mathrm{~m} / \mathrm{sec}$, and the mobile robot backward speed appears $0.6046 \mathrm{~m} / \mathrm{sec}$ in reality, while the virtual mobile robot backward speed being adjusted the speed parameter reveals $81.09 \mathrm{~m} / \mathrm{sec}$, about $0.6046 \mathrm{~m} / \mathrm{sec}$. The robot arm moves upward speed about $0.0227 \mathrm{~m} / \mathrm{sec}$ in reality, while the virtual robot arm forward speed being adjusted the speed parameter reveals $0.00789 \mathrm{~m} / \mathrm{sec}$, about $0.0227 \mathrm{~m} / \mathrm{sec}$, and the robot arm backward speed appears $0.0241 \mathrm{~m} / \mathrm{sec}$ in reality, while the virtual mobile robot backward speed being 
adjusted the speed parameter reveals $0.00845 \mathrm{~m} / \mathrm{sec}$, about $0.0241 \mathrm{~m} / \mathrm{sec}$. The detailed data are shown in Table 1.

The follow-up adjustment of speed parameter reduces the operation errors between VR and the real robot down to the least so that the movement and assembly of the robot in VR and the solid robot are almost synchronous. Furthermore, the wireless remote control assists in successfully controlling the robot in reality to complete the assembly, as shown in Fig. 10.

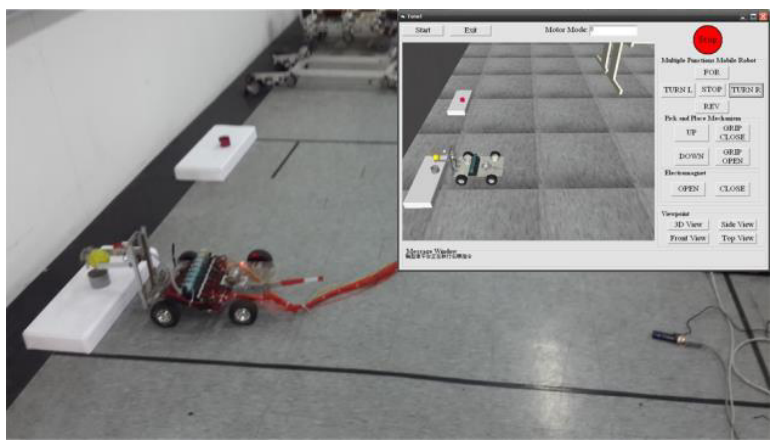

Fig. 10. Experimental procedure.

Table 1. Mobile robot speed parameter relative speed in virtual and reality.

\begin{tabular}{|c|c|c|}
\hline & $\begin{array}{l}\text { Virtual reality robot } \\
\text { speed } \\
\quad(\mathrm{m} / \mathrm{sec})\end{array}$ & $\begin{array}{c}\text { Reality mobile } \\
\text { robot speed }(\mathrm{m} / \mathrm{sec})\end{array}$ \\
\hline $\begin{array}{l}\text { mobile robot } \\
\text { forward }\end{array}$ & 77.84 & 0.5804 \\
\hline $\begin{array}{l}\text { mobile robot } \\
\text { backward }\end{array}$ & 81.09 & 0.6046 \\
\hline Pick-up & 0.00793 & 0.0227 \\
\hline Pick-down & 0.00845 & 0.0241 \\
\hline $\begin{array}{l}\text { Gripper } \\
\text { close }\end{array}$ & $22.0327(\mathrm{deg} / \mathrm{sec})$ & $0.3845(\mathrm{rad} / \mathrm{sec})$ \\
\hline Gripper open & $21.7468(\mathrm{deg} / \mathrm{sec})$ & $0.3796(\mathrm{rad} / \mathrm{sec})$ \\
\hline
\end{tabular}

\section{Conclusions}

EON is utilized for real-time connection control of solid robot to automatically complete the pick and place tasks in VR. The past research mostly reduced the errors by improving the transmission speed after the camera transmitting the on-site images. In this study, VR, replacing a camera, revises the speed parameter of the robot for synchronism between virtual and real frames. Moreover, simulation tree in EON is applied to presenting the real physical properties of an object, such as the moving speed and collision of a mobile robot and arm. When such physical properties are wrongly or not set, the simulation in VR would be largely reduced. For example, when the robot touches the work platform without stopping or even passing it through, the plug-in assembly would not be completed.

In the real operation, it requires several attempts and corrections to grab the plug-in and successfully complete the assembly. VR allows freely changing the angles of view to make the grab and assembly be more easy. A new function to show the information of coordinate, moving distance, and rotation angle of the robot or other objects in VR frames could have the users more clearly understand the situation in the field. Or, including a virtual guidance could assist the users more easily operation the process, which are considered as a different research domain.

The fraction that this search needs to be strengthened which explained as follows: To raise the robot feedback information of HMI, that can be provided for user to make references. Because of many bugs happening while testing, Bluetooth v2.1+EDR is used for VR with PLC may be not so suitable.

\section{References}

1. F.C. Hsu, VR-Based Teleoperation System for Industrial Robots, Master's thesis, Department of Mechanical Engineering, Chung-Yuan Christian University (2000)

2. Y.H. Huang, Applying Virtual Guidance for Robot Teleoperation, Master's thesis, Department of Electrical and Control, National Chiao Tung University (2007)

3. S.W. Chuang, Virtual Assembly Modeling, Master's thesis, Department of Mechanical Engineering, National Central University (1997)

4. T.J. Ke, Personal Computer Assembly by Virtual Reality Technique, Master's thesis, Department of Computer Science, National Chiao Tung University (1998)

5. E.A. Orady, T.A. Osman, 21st International Conference on Computers and industrial Engineering, 33, pp. 87-90, (1997)

6. T.A. Chen, Development of Robotic Gripper for Telepresence in a Telerobotic System, Master's thesis, Department of Electrical and Control Engineering, National Chiao Tung University (2003)

7. H.B. Chen, Controller Design and Virtual Reality of Tatung TTUP3D Parallel Robot, Master's thesis, Department of Mechanical Engineering, Tatung University (2001)

8. E. Freund, J. RoBmann, Proceedings of the 2001 IEEE International Conference on Robotics and Automation, pp. 21-26, (2001)

9. X. Xie, L. Sun, Z. Du, Z. Zong, Proceedings of the 6th World Congress on Intelligent Control and Automation, pp. 21- 23, (2006)

10. Y.C. Lin, Virtual Assembly Modeling, Master's thesis, Department of Mechanical Engineering, National Central University (2002) 
11. D.Y. Chiou, Object-oriented System Modeling for Web-besed Telerobot Systems with a Real Implication, Master's thesis, Institute of Industrial Engineering, National Taiwan University (2000)

12. Imre Cikajlo, Journal of Medical and Biological Engineering, 34, 4, pp. 319-326, (2014)

13. C.W. Yin, N.Y. Sien, L.A. Ying, S.F. Chung and D.T. Leng, Clinical Rehabilitation, 28, 11, pp. 11071114, (2014)

14. Junji Nomura and Kazuya Sawada, Annual Reviews in Control, 25, pp. 99-109, ( 2001)

15. H. Brenton, J. Hernandez, F. Bello, P. Strutton, S. Purkayastha, T. Firth, and A. Darzi, Computers \& Education, 49, 1, pp. 32-53, (2007)

16. A. Jönsson, J. Wall, G. Broman, International Journal of Machine Tools \& Manufacture, 45, pp. 795-801, (2005) 